\title{
Vibration Signal Parameter Estimation in Variable Speed: Algorithms and performance bounds
}

\author{
Ali Abbadi, Cécile Capdessus, Karim Abed-Meraim, and Edgard Sekko
}

\begin{abstract}
Vibration signal parameter estimation for rotating machinery diagnostics operating under variable speed conditions is considered. At first, we provide a brief survey of existing methods for Quadratic Phase Signal (QPS) parameter estimation. Then, we introduce improved solutions for the general QPS case and the Order QPS (O-QPS) case, respectively. For all considered cases (namely the QPS, O-QPS with tachometer and O-QPS without tachometer), we develop the Cramer Rao Bounds to assess and compare the estimation performance limits for each model. Finally, we compare the performance of all considered methods and highlight, in particular, the gain of the proposed solutions.
\end{abstract}

Keywords-Cramer-Rao Bound (CRB), Higher order Ambiguity Function (HAF), Quadratic Phase Transform (QPT), Variable Speed, Vibration Signal.

\section{INTRODUCTION}

In the last decades, vibration signal analysis techniques have attracted increasing attention and became a hot research topic in the rotating machinery diagnostics area thanks to its potential advantage over invasive diagnostics techniques.

It is based on the recording, via accelerometers or other modern technology sensors (e.g. high-speed laser sensors), of the vibration levels and frequencies of the machinery of interest and then using that signal to analyze how healthy the machine's components are. The vibration signal analysis is the realm of developing appropriate models, methods and algorithms which help determining the health of the machine and identify possible impending problems like unbalance, misalignment, looseness, lubrication issues, etc. Indeed, based on a physical model of the vibration signal, and taking into account shocks, friction, rotation speed, structural resonance and propagation, relevant indicators can be elaborated to monitor rotating machinery. Such a non invasive analysis has been shown to be very efficient to detect problems such as $[1,2]$ : Bearing failures, Imbalance, Resonance and natural frequencies, Gearbox failures, Electrical motor faults, etc.

Now, the introduction of artificial intelligence techniques for automating the diagnosis implies the development of appropriate methods that can be implemented in all operating conditions, even in the cases where classical approaches fail, such as in time varying operating conditions. In that case, unlike the conventional diagnostic approach based on stationary assumption, the non stationary assumptions open a way to the diagnosis of the rotating machinery under variable speed conditions.

Manuscript received March 28, 2021;revised June 4, 2021. Ali Abbadi is with Ecole Supérieure Ali Chabati, Réghaia, Algiers, AlGERIA. (e-mail: abbadi.ali.khaled@gmail.com)

Cécile Capdessus, Karim Abed-Meraim and Edgard Sekko are with PRISME laboratory, University of Orléans, 8 Rue Léonard de Vinci, 45072, Orléans, France. (e-mails: firstname.name@univ-orleans.fr)

Digital Object Identifier (DOI): 10.53907/enpesj.v1i1.19
Based on time varying signal analysis tools for rotating machinery diagnosis under variable speed operation, three classes of order tracking have been proposed and broadly discussed in the literature [3-6], which are the resampling based methods, the Kalman filter based methods (tracking methods) and the transform based methods. The resampling based methods transform the signal data from the time domain to the angular (order) domain to obtain a sampled signal at constant angular increment instead of a sampled signal at constant time increment. They result in limited order resolution related to the constant increment interval and a finite number of samples. In order to improve the accuracy of order tracking the Kalman filter based methods were introduced. They consist in recursively minimizing a cost function for parameter estimation of time-varying frequencies to overcome many of the limitations of order resolution and allow the optimal estimation of the amplitude and phase of an order. Unfortunately, they are computationally expensive and require prior knowledge of some information such as the number of orders (frequencies) to be extracted, needed for Kalman filter initialization and convergence. These disadvantages limit the use of these methods to experienced operators and in offline conditions contrary to the robust and rather easy-to use transform methods. The transform methods are based on the modification of the DFT (Discrete Fourier Transform) kernel to a time varying kernel. They are both the easiest to use, and the simplest to implement. These methods present some drawbacks related to certain resolution limits and for some of them the need to add a tachometer signal as reference regime.

In this paper, we will focus on this last class of methods where the data can be analyzed and processed with many techniques, which are briefly reviewed below. The basic principle of these transforms is the replacement of the classical kernel of Fourier transform by a kernel whose base functions have frequencies that are proportional to the rotation speed of the system to be monitored. A drawback of this modification is that the kernel functions do not constitute any more an orthonormal basis. The Speed Transform (ST), proposed by Capdessus et al. in [7, 8], is normalized with the integration interval, which ensures asymptotic orthogonality in the case of linear speed variations. To cope with a wider range of speed variations, the Time Variant Discrete Fourier Transform (TVDFT), proposed by Blough et 
al. in $[3,4,9]$, comprises an orthogonality compensation matrix. But the most general technique is the Velocity Synchronous Discrete Fourier Transform (VSDFT) proposed by Borghesani et al. in $[5,6,10]$, which consists in applying the time-variant kernel Fourier transform to a corrected temporal signal. The correction, which is a mere product of the raw signal by the angular speed, ensures the orthogonality of the decomposition whatever the speed variations.

In the case of transform based methods like ST [7], prior knowledge of the rotation frequency variations is needed and this information is provided by a tachometer*; hence only the order of the signal harmonics has to be estimated. However, the tachometer information is not always available, in which case techniques that operate without such prior knowledge are required. Interestingly, these time-varying kernel Fourier transforms are linked with the Quadratic Phase Transform (QPT) in that they fit to Polynomial Phase Signals (QPS). The QPS has been already considered in many signal processing applications including power systems [11], radar [12], and geophysics [13].

In this paper, we will extend the works in $[14,15]$. Several methods for polynomial phase signal analysis already exist in the literature. We first provide an overview of the existing algorithms for polynomial phase parameter estimation which includes the time-frequency based methods in [16,17], the (higher order) ambiguity function based methods in $[18,19]$, the iterative methods in [20,21], and the fast decomposition methods in [22,23]. Note that this survey is mainly limited to methods introduced in the signal processing and communications community that deserve to be better known and recognized as they provide interesting extensions to the existing tools used in vibration signal analysis.

The proposed extended work consists of the previously mentioned survey of existing methods for QPS parameter estimation followed by two new methods that lead to improved estimation accuracy with reduced computational cost. Then, we derive the CRB for the considered data models with or without a tachometer information and provide a comparative performance analysis via simulation experiments.

\section{Problem Formulation}

Rotating machine with constant speed are characterized by harmonic vibration signal of the form [9]

$$
x(n)=\sum_{p=1}^{P} A_{p} e^{j 2 \pi f_{p} n}+w(n)
$$

where $P$ is the number of modes (harmonics), while $A_{p}$ and $f_{p}$ are the complex amplitude and the frequency of the $p t h$ mode. In this case, the signal is referred to as a linear phase one (i.e. the component's phase terms vary linearly with time $\left.\phi_{p}(n)=2 \pi f_{p} n\right)$. The additive noise $w(n)$ is assumed to be of zero-mean, white and gaussian distributed with variance $\sigma_{w}^{2}$.

In the case of variable speed, the signal phase would depend non-linearly with time and the model becomes $[7,8]$

$$
x(n)=\sum_{p=1}^{P} A_{p} e^{j \phi_{p}(n)}+w(n)
$$

${ }^{*}$ Recall that a tachometer is an instrument measuring the rotation speed and provides prior knowledge of the instantaneous fundamental frequency. $\phi_{p}(n)$ being the phase term of the $p t h$ component.

A particular case of interest, widely considered in the literature, is the one with uniform angular acceleration that corresponds to multi-component chirp signal. This case may be encountered in two different situations. First, on test benches where part of the vibrations measurements are done during a run-up or a coast-down of the machine. One can mention as an example the run-up and run-down experiments performed on gears, turbofans, or thermal motors, and other systems in order to study their stability and their balance or determine their critical speeds [24-28]. These techniques are now classically provided in commercial test devices and are performed through a linearly varying speed scenario. Second, in real-life operation, though the speed variations generally cannot be assumed to be linear, yet during the start-up and the coast-down stages, there usually is a portion of the speed variations that can still be approximated linearly [29]. In that context, the signal model is given by

$$
x(n)=\sum_{p=1}^{P} A_{p} e^{j 2 \pi\left(f_{p} n+\gamma_{p} n^{2}\right)}+w(n)
$$

As we can see, the study of this signal coincide with the wellknown polynomial phase signal analysis $[22,30]$.

In this paper, we focus only on the second order case (i.e. Quadratic phase signal). Therefore, our first objective is to study the parameter estimation problem for the signal model in (3). More precisely, after a brief survey of the methods in the literature, we propose to refine one of them (namely the FQPT method in [22]) and to compare it to the other existing techniques to assess its performance.

Our second objective, consists of considering a particular case of QPT signals given by the model

$$
x(n)=\sum_{p=1}^{P} A_{p} e^{j 2 \pi o_{p}\left(f n+\gamma n^{2}\right)}+w(n)
$$

where $o_{p}$ is referred to as the $p t h$ harmonic order. Parameters $(f, \gamma)$ might be known if a tachometer is available or unknown otherwise. In the latter case, we have to assume that $o_{1}=1$ (corresponding to the fundamental frequency) and $o_{p}>1$ for $p \geq 2$, otherwise the model is not uniquely identifiable*.

The model in (4) has been shown to well represent the vibration signal in presence of 'faults' or 'abnormal' behavior of the machine [7,9]. In this case, the harmonic parameters are used to 'control' and diagnose the current status of the rotating machine. For this particular model, we propose to adapt the HAF (Higher order Ambiguity Function) method to extract the desired signal parameters.

Finally, we propose to compare the two models in (3) and (4) through the evaluation of their corresponding Cramer-Rao Bounds.

*Indeed, the two sets of parameters $\left\{f, \gamma, o_{p}, p=1, \cdots, P\right\}$ and $\left\{o_{1} f, o_{1} \gamma, o_{p} / o_{1}, p=1, \cdots, P\right\}$ lead to the same observation $x(n)$ and hence, without this normalization, one cannot identify our parameters in a unique way. 


\section{OVERVIEW OF MAJOR QPS PARAMETER ESTIMATION ALGORITHMS}

Polynomial phase signals have been used in many signal processing applications to model different physical phenomena characterized by nonlinear phases. We consider here the case of quadratic phase signals corresponding to the second order polynomial phase model given in (3). The signal being gaussian distributed, the maximum likelihood (ML) estimation of the QPS parameters coincides with the nonlinear least squares problem

$$
\min _{\Theta} \sum_{n=0}^{N-1}\left|x(n)-\sum_{p=1}^{P} A_{p} e^{j 2 \pi\left(f_{p} n+\gamma_{p} n^{2}\right)}\right|^{2}
$$

where $\Theta$ is the vector of unknown QPS model parameters. This optimization problem being highly nonlinear and complex, many suboptimal solutions have been developed in the literature and are briefly reviewed in this section.

\section{A. Fast Quadratic Phase Transform (FQPT) method}

The FQPT that was developed by Ikram et al. in [22], is based on the quadratic phase transform (QPT) for joint phase parameter estimation of multi-component chirp signals. The QPT is a second order polynomial phase transform. It has the ability to analyze chirp signals in the same way as Fourier transform analyzes sinusoidal signals. The Discrete QPT (DQPT) is defined as

$$
D Q P T\{x(n)\}=X(k, l)=\sum_{n=0}^{N-1} x(n) e^{-j 2 \pi\left(\frac{k}{N} n+\frac{l}{M} n^{2}\right)}
$$

where $k=0, \cdots, N-1$ and $l=0, \cdots, M-1$.

The FQPT is a fast implementation of the DQPT that transforms the one-dimensional sequence $x(n)$ into a two-dimensional sequence $X(k, l)$. It proceeds to joint phase parameters estimation after signal dechirping search. It uses some symmetry and translation characteristics to perform a fast transform and provide a significant computational saving. The FQPT can be seen as an approximate maximum likelihood method for large data sample.

Note that once the phase parameters are estimated from the peaks location of the DQPT, the amplitude coefficients are obtained by a simple least squares optimization according to

$$
\left[\begin{array}{c}
\hat{A}_{1} \\
\vdots \\
\hat{A}_{P}
\end{array}\right]=\mathbf{M}^{\#}\left[\begin{array}{c}
x(0) \\
x(1) \\
\vdots \\
x(N-1)
\end{array}\right]
$$

where $\mathbf{M}^{\#}$ refers to the pseudo-inverse of matrix $\mathbf{M}$ which is given by

$$
\left[\begin{array}{ccc}
1 & \cdots & 1 \\
e^{j 2 \pi\left(\hat{f}_{1}+\hat{\gamma}_{1}\right)} & \cdots & e^{j 2 \pi\left(\hat{f}_{P}+\hat{\gamma}_{P}\right)} \\
\vdots & & \vdots \\
e^{j 2 \pi\left(\hat{f}_{1}(N-1)+\hat{\gamma}_{1}(N-1)^{2}\right)} & \cdots & e^{j 2 \pi\left(\hat{f}_{P}(N-1)+\hat{\gamma}_{P}(N-1)^{2}\right)}
\end{array}\right]
$$

Typically, $M \gg N$ since the value of the second order coefficient is usually much smaller than the first order coefficient and hence requires a finer grid search.

\section{B. Higher Order Ambiguity Function (HAF) method and its variants}

The conventional HAF method, first introduced by Peleg et al. in [31] as the so called Discrete Polynomial Phase Transform (DPT) and named later HAF by Porat in [32], is a recursive algorithm that finds the highest-order phase coefficient, removes its contribution from the signal and repeats the process until all required phase parameters have been estimated. For a monocomponent QPS, $x(n)=A e^{j 2 \pi\left(f n+\gamma n^{2}\right)}$, the basic HAF is defined as follows

$$
\begin{aligned}
& Q P T[x(n), \tau]=x(n+\tau) x(n-\tau)^{*}=|A|^{2} e^{j 2 \pi 2 f \tau} e^{j 2 \pi 4 \gamma \tau n} \\
& \operatorname{HAF}(\omega)=\operatorname{DTFT}\{Q P T[x(n), \tau]\} \\
& =\sum_{n=0}^{N-1} Q P T[x(n), \tau] e^{-j \omega n}
\end{aligned}
$$

The highest order $\gamma$ is estimated as

$$
\hat{\gamma}=\frac{1}{8 \tau \pi} \arg \max _{\omega}|H A F(\omega)|
$$

Once the highest order $\hat{\gamma}$ is estimated, we proceed to the suppression of its contribution by demodulation as

$$
z(n)=x(n) e^{j\left(-2 \pi \hat{\gamma} n^{2}\right)} \approx A e^{j 2 \pi f n}
$$

The lowest order $f$ is estimated as

$$
\hat{f}=\frac{1}{2 \pi} \arg \max _{\omega}|\operatorname{DTFT}\{z(n)\}|
$$

For the multi-component case, the transform in (8) would provide $P$ sinusoids (plus non desired cross terms) that can be estimated using DTFT or high resolution methods like MUSIC or ESPRIT [20]. The dechirping in (11) is then achieved successively for $\hat{\gamma}_{1}, \cdots, \hat{\gamma}_{P}$ for the estimation of the first order parameters $f_{p}, p=1, \cdots, P$.

Unfortunately, HAF-based methods are characterized by the use of matched filter that suffer from an identifiability problem when dealing with multiple component QPS's having the same highest order phase coefficients.

To overcome this problem a so called product higher-order ambiguity function (PHAF) was developed, by Barbarossa et al. in [18]. The PHAF algorithm combines HAF and HIM (High order Instantaneous Moment) techniques for the detection and parameter estimation of multi-component QPS impinged in white Gaussian noise. It is defined as the product of $L$ HAFs for $L$ sets of lag parameters, and formulated as follows

$$
\operatorname{PHAF}(\omega)=\prod_{l=1}^{L} \operatorname{HAF}^{(l)}(\omega)
$$

where $\operatorname{HAF}^{(l)}(\omega)=\sum_{n=0}^{N-1} Q P T\left[x(n), \tau^{(l)}\right] e^{-j \omega F^{(l)} n}, \tau^{(l)}$ refers to the $l$ th lag term, and $F^{(l)}=\frac{\tau^{(l)}}{\tau^{(1)}}$ is a frequency scaling factor. The highest order $\gamma$ is estimated as

$$
\hat{\gamma}=\frac{1}{8 \tau^{(1)} \pi} \arg \max _{\omega}|\operatorname{PHAF}(\omega)|
$$


The use of the PHAF offers a number of advantages over the HAF. It solves the identifiability problem, improves noise rejection capabilities, and leads to improved performance as compared to HAF method.

Another improved version introduced in the literature by Ikram et al. in [20,21], is the iterative HAF method.

It is based on iterative calculation of the polynomial phase signal coefficients. First, the second-order phase parameter is iteratively estimated and updated using a quadratic transform with appropriate lag parameter in each iteration in order to improve the estimation accuracy. Then the first-order phase parameter is estimated based on the same transform technique of demodulated signal, as defined below

$z_{q}(n)=z_{q-1}\left(n+\tau_{q+1}\right) z_{q-1}^{*}(n), \quad n=0, \cdots, N-\tau_{q-1}-1$

where $z_{q-1}(n)$ is the demodulated signal defined as $z_{q-1}(n)=$ $x(n) e^{-j \hat{\gamma}_{q-1} n^{2}}, n=0, \cdots, N-1$ with $q$ is the iteration index and $z_{0}(n)=x(n)$.

Each estimated phase parameter is then refined using MUSIC or other high resolution algorithms. It is shown in [20], that the iterative HAF leads to a significant performance gain, especially at low SNRs.

Remark: A main advantage of HAF-type methods is their ability to deal with polynomial phase signals of any order (see [23,30-32] for details).

Therefore, in the case of non-linear variable speed with nonuniform angular acceleration, one can use polynomial phase models of order higher than two and estimate their parameters via the considered HAF-type methods. Note that any continuous function over a finite interval can be 'well approximated' by a polynomial function as shown in [33]. However, the estimation cost of the phase parameters increases significantly with the polynomial order and hence using a second order polynomial represents a good trade-off in many practical situations [24, 25, 27].

\section{Time-Frequency Distribution (TFD) based method}

This algorithm was developed, by Barkat et al. in [16, 17], to select and extract separately all components of frequency modulated signal. It's based on joint time-frequency analysis of the signal. Firstly, a one dimensional signal is transformed to TimeFrequency (TF) domain using appropriate reduced interference TF kernel (in $[16,17]$, the B-distribution was used).

Then, the undesired low energy peaks are removed by noise thresholding. After that, the number of components is estimated as the number of peaks of the TFD slice by means of the maximum argument of the histogram of the number of peaks computed for each time instant. Finally, a components separation procedure is applied to extract separately all the components by finding the components frequencies as the peaks positions of the TFD slice and identifying the crossing components using a smallest distance criterion.

This algorithm proved its superiority over the HAF method for the estimation of a multi-component signal.
The algorithm does not use any prior information about the various components to be extracted and its performance depends on the ability to suppress the cross-terms and needs high time-frequency resolution. Unfortunately, the algorithm needs a distribution that can reveal the features of the signal as clearly as possible to have a clean TFD, and has expensive computational load due to TFD computation and 2D search.

Note that, chirp signals are represented by 'straight lines' in the TF domain. Hence the problem of chirp parameter estimation has been assimilated to a contour (straight lines) extraction problem in an image and dedicated image processing tools have been used in [34] for solving this particular estimation problem. Other works related to the use of TF signal analysis tools for vibration signals can be found in $[35,36]$.

\section{Proposed Algorithms}

We introduce in this section an improved version of the FQPT referred to as the Refined FQPT (R-FQPT) as well as adapted versions of the HAF method to deal with the 'order' estimation problem related to the model in (4).

\section{A. R-FQPT Algorithm}

The FQPT, developed by Ikram and al. in [22], is described by

$$
Q P T\{x(n)\}=X(k, l)=\sum_{n=0}^{N-1} x(n) e^{-j 2 \pi\left(\frac{k}{N} n+\frac{l}{M} n^{2}\right)}
$$

where $N$ is the number of samples and $M \geq N^{2}$. The fast computation of the FQPT is based on three properties (see [22] for more details)

- Decomposition of the QPT in multiple DFT:

$$
\begin{aligned}
& z^{(l)}(n)=x(n) e^{-j 2 \pi \frac{l}{M} n^{2}} \\
& X(k, l)=\operatorname{DFT}_{n}\left(z^{(l)}(n)\right)
\end{aligned}
$$

- Decimation : used by Fast Fourier transform FFT to compute with reduced computational cost the Discrete Fourier Transform (DFT):

$$
\begin{aligned}
& D F T_{m}\left(z^{(l)}\left(2^{r} m+s\right)\right)=D F T_{m}\left(z^{(l)}\left(2^{r+1} m+s\right)\right) \\
& +e^{-j 2 \pi\left(\frac{k}{N} 2^{r}\right)} D F T_{m}\left(z^{(l)}\left(2^{r+1} m+s+2^{r}\right)\right)
\end{aligned}
$$

- Symmetry:

$$
\begin{aligned}
D F T_{m}\left(z^{\left(l+\frac{M}{2^{r+1}}\right)}\left(2^{r} m+s\right)\right)= & \operatorname{DFT}_{m}\left(z^{(l)}\left(2^{r} m+s\right)\right) \\
& . e^{-j 2 \pi\left(\frac{s^{2}}{2^{r+1}}\right)}
\end{aligned}
$$

By using the previous properties, it is shown in [22] that the computational cost can be reduced from $O\left(N^{2} M\right)$ to $O(N M)$ flops. This cost is still relatively high and increases with the 2D grid size $N$ and $M$. Therefore to preserve 'a moderate' numerical complexity, one should limit the number of grid points which unfortunately affects the estimation precision of the FQPT. To overcome this drawback, we propose in the following a new 
version of FQPT that refines the parameter estimation through a two-step procedure.

For that, we will use a modified filtering/unwrapping algorithm to refine the initial parameter estimate $\left(\hat{f}^{(0)}, \hat{\gamma}^{(0)}\right)$ given by the FQPT algorithm. First, we demodulate the received quadratic polynomial phase signal using the initial FQPT estimated parameters according to $y(n)=x(n) e^{-j 2 \pi\left(\hat{f}^{(0)} n+\hat{\gamma}^{(0)} n^{2}\right)}$. Then, we apply a low-pass filtering to enhance the SNR. After that, we create a vector $\mathbf{v}=[v(0), v(1), \cdots, v(Q-1)]^{T}$ of unwrapped phase which is modeled as a new polynomial phase signal (i.e. $\left.v(k) \approx \varphi+\delta f\left(k w_{s}\right)+\delta \gamma\left(k w_{s}\right)^{2}\right)$ in noise with $Q=\frac{N}{w_{s}}, \omega_{s}$ being a down-sampling factor used to reduce the cost and improve the resolution of the method (this is needed here since the residual parameters $\delta f$ and $\delta \gamma$ are close to zero). Then, we apply a standard least squares estimation to obtain the new unknown phase parameters vector, defined as $\mathbf{v}=\left[\varphi, w_{s} \delta f, w_{s}^{2} \delta \gamma\right]^{T}$, according to

$$
\begin{aligned}
& \arg \min _{\mathbf{v}} \sum_{k=0}^{Q-1}\left|v(k)-\varphi+\delta f\left(k w_{s}\right)+\delta \gamma\left(k w_{s}\right)^{2}\right|^{2} \\
& =\left[\begin{array}{ccc}
1 & 0 & 0 \\
1 & 1 & 1 \\
\vdots & \vdots & \vdots \\
1 & (Q-1) & (Q-1)^{2}
\end{array}\right]^{\#}\left[\begin{array}{c}
v(0) \\
v(1) \\
\vdots \\
v(Q-1)
\end{array}\right]
\end{aligned}
$$

where \# refers to the matrix pseudo-inverse,

Finally, we obtain refined phase parameters by accumulating each FQPT initial estimated phase parameters with corresponding estimated phase parameters deviations according to $\hat{f}=\hat{f}^{(0)}+\delta \hat{f}$ and $\hat{\gamma}=\hat{\gamma}^{(0)}+\delta \hat{\gamma}$.

\section{B. Adapted HAF methods for order QPS}

For the case of O-QPS model of (4), the two steps of the HAF method are not needed (since, for each signal component, we have only one parameter to estimate instead of two for the general QPS model). Therefore, if a tachometer is available, the HAF method consists of only one step according to:

- For a given lag time $\tau$, compute signal

$$
\begin{aligned}
y(n)=x(n+\tau) x(n)^{*} & =\sum_{p=1}^{P}\left|A_{p}\right|^{2} e^{j \phi_{p}(\tau)} e^{j 4 \pi \tau \gamma o_{p} n} \\
& + \text { cross terms + noise }
\end{aligned}
$$

with $\phi_{p}(\tau)=2 \pi o_{p}\left(f \tau+\gamma \tau^{2}\right)$. Since the orders $o_{p}$ are typically in the range $[1,10]$, a proper choice for time lag $\tau$ corresponds to $2 \tau|\gamma| \approx 0.1$ so that the frequency terms $\left|4 \pi \tau \gamma o_{p}\right|$ are maximally separated while not exceeding the upper limit $2 \pi$ to avoid estimation ambiguity problems.

- Apply ESPRIT algorithm to signal $y(n)$ for the high resolution estimation of the $P$ pulsation terms $\hat{\omega}_{p} \approx 4 \pi \tau \gamma o_{p}$ or equivalently

$$
\hat{o}_{p}=\hat{\omega}_{p} /(4 \pi \tau \gamma)
$$

- Eventually, apply the iterative procedure of [37] for the refining of the previous parameter estimate.
If the tachometer is not available, parameter $\gamma$ can be estimated by using the fact that $o_{1}=1$ so that the first (smallest) pulsation value would correspond to $\gamma$, i.e.

$$
\hat{\gamma}=\hat{\omega}_{1} /(4 \pi \tau)
$$

Once $\gamma$ obtained, parameter $f$ can be estimated via (12) with $z(n)=x(n) e^{-j 2 \pi \hat{\gamma} n^{2}}$.

\section{CRAMER-RaO Bounds DERIVATION}

We provide here the detailed derivation of the CRB for the statistical models given in section II. Recall that, for a given data model depending on an unknown parameter vector, the CRB provides the lower limit to the statistical error variance for the unbiased estimation of the unknown model parameters.

The CRB is computed as the inverse of the Fisher Information Matrix (FIM) which entries are given by

$$
[F I M]_{i, j}=-E\left[\frac{\partial^{2} \ln (p(\mathbf{x} ; \Theta))}{\partial \theta_{i} \partial \theta_{j}}\right]
$$

where $E[$.$] represents the statistical expectation operator, p(\mathbf{x} ; \Theta)$ denotes the likelihood function, $\mathbf{x}$ is the observation vector and $\Theta$ denotes the parameter vector to be estimated.

The error covariance matrix of any unbiased estimator $\hat{\xi}$ of parameter vector $\xi=\mathbf{g}(\Theta)$ (denoted $\operatorname{Cov}(\hat{\xi})$ ), where $\mathbf{g}(\Theta)$ is a locally differentiable function, is lower bounded by the CRB according to (i.e. $\operatorname{Cov}(\hat{\xi})-C R B(\xi)$ is a positive semi-definite matrix)

$$
\operatorname{Cov}(\hat{\xi}) \geq C R B(\xi)=\nabla \mathbf{g}(\Theta) C R B(\Theta)(\nabla \mathbf{g}(\Theta))^{T}
$$

where $C R B(\Theta)=[F I M]^{-1}$ and $\nabla \mathbf{g}(\Theta)$ is the gradient matrix which $(i, j)^{t h}$ element is defined by $\frac{\partial g_{i}(\Theta)}{\partial \theta_{j}}$. In particular, this means that the error variance in the estimation of parameters $g_{i}(\Theta)$ is greater than the $i-t h$ diagonal element of $C R B(\xi)$.

Our case of interest corresponds to the Gaussian CRB for which the FIM matrix can be expressed by the Slepian-Bang formula [32]

$$
[F I M]_{i, j}=-E\left[\frac{\partial^{2} \ln (p(\mathbf{x} ; \Theta))}{\partial \theta_{i} \partial \theta_{j}}\right]=\frac{2}{\sigma_{w}^{2}} \operatorname{Re}\left\{\frac{\partial \mathbf{s}^{H}}{\partial \theta_{i}} \frac{\partial \mathbf{s}}{\partial \theta_{j}}\right\}
$$

where s denotes the noiseless QPS signal in (3) and Re [.] refers to the real part of a complex number. Note that in (24), we do not include the unknown parameter $\sigma_{w}^{2}$ in the parameter vector $\Theta$ since the FIM of the desired QPS parameters is decoupled from the noise variance [32].

In the sequel, we provide the FIM expressions for the three parametric models given in section II.

(a) General QPS case: The observed signal model is given by

$$
s(n)=\sum_{p=1}^{P} \rho_{p} e^{i \varphi_{p}} e^{2 \pi i\left(f_{p} n+\gamma_{p} n^{2}\right)}
$$

where the complex amplitudes are written as $A_{p}=\rho_{p} e^{i \varphi_{p}}$. In that case, the unknown parameter vector of size $4 P \times 1$ 
is given by $\Theta=\left[\left\{\rho_{p}\right\},\left\{\varphi_{p}\right\},\left\{f_{p}\right\},\left\{\gamma_{p}\right\}\right]^{T}$ and the FIM is expressed as

$$
F I M=\frac{2}{\sigma_{w}^{2}} \operatorname{Re}\left\{\tilde{\mathbf{S}}^{H} \tilde{\mathbf{S}}\right\}
$$

where the $N \times(4 P)$ matrix $\tilde{\mathbf{S}}$ is given by

$$
\tilde{\mathbf{S}}=\left[\left\{\frac{\partial \mathbf{s}}{\partial \rho_{p}}\right\},\left\{\frac{\partial \mathbf{s}}{\partial \varphi_{p}}\right\},\left\{\frac{\partial \mathbf{s}}{\partial f_{p}}\right\},\left\{\frac{\partial \mathbf{s}}{\partial \gamma_{p}}\right\}\right]
$$

(b) Order QPS case without tachometer: In that case, the observed signal model is given by

$$
s(n)=\sum_{p=1}^{P} \rho_{p} e^{i \varphi_{p}} e^{2 \pi i o_{p}\left(f n+\gamma n^{2}\right)}
$$

with $o_{1}=1$. For this model, we have $\Theta=$ $\left[\left\{\rho_{p}\right\},\left\{\varphi_{p}\right\},\left\{o_{p}\right\}_{p>1}, f, \gamma\right]$ and the FIM is as in (26) with

$$
\tilde{\mathbf{S}}=\left[\left\{\frac{\partial \mathbf{s}}{\partial \rho_{p}}\right\},\left\{\frac{\partial \mathbf{s}}{\partial \varphi_{p}}\right\},\left\{\frac{\partial \mathbf{s}}{\partial o_{p}}\right\}_{p>1},\left\{\frac{\partial \mathbf{s}}{\partial f}\right\},\left\{\frac{\partial \mathbf{s}}{\partial \gamma}\right\}\right]
$$

For parameters $f_{p}=o_{p} f=g_{p}(\Theta)$, and $\gamma_{p}=o_{p} \gamma=$ $h_{p}(\Theta)$ the minimum error variances are computed as

$$
\begin{aligned}
& C R B\left(f_{p}\right)=\nabla g_{p}(\Theta) C R B(\Theta)\left(\nabla g_{p}(\Theta)\right)^{T} \\
& C R B\left(\gamma_{p}\right)=\nabla h_{p}(\Theta) C R B(\Theta)\left(\nabla h_{p}(\Theta)\right)^{T}
\end{aligned}
$$

(c) Order QPS case with tachometer: The observed signal model is like in (28) but with parameters $f$ and $\gamma$ known. In that case, the unknown parameter vector becomes $\Theta=\left[\left\{\rho_{p}\right\},\left\{\varphi_{p}\right\},\left\{o_{p}\right\}\right]$ and the matrix $\tilde{\mathbf{S}}=\left[\left\{\frac{\partial \mathbf{s}}{\partial \rho_{p}}\right\},\left\{\frac{\partial \mathbf{s}}{\partial \varphi_{p}}\right\},\left\{\frac{\partial \mathbf{s}}{\partial o_{p}}\right\}\right]$.

\section{PeRformance ANALYSis AND COMPARATIVE RESUlts}

In this section, numerical investigations are conducted for the following objectives:

- For the general QPS case, we provide a comparative performance analysis of the different methods considered in the survey part. All results are compared to the CRB to assess their statistical efficiency.

- The previous comparative study is used to highlight the performance gain of the proposed R-FQPT method in the moderate and low SNR region.

- We compare the CRBs for the general QPS, O-QPS with tachometer, and O-QPS without tachometer cases. In particular, this comparison allows us to evaluate the potential performance gain brought by the tachometer use.

- We assess the performance of the proposed HAF method adapted to the O-QPS case and compare it to the corresponding CRB.
- Finally, the R-FQPT is compared to ST and VSDFT on a simulated vibration signal.

We assume that the observed vibration signal is a multicomponent QPS in additive white Gaussian noise. The additive noise is randomly generated as circular complex white Gaussian process with zero mean and unknown variance $\sigma_{w}^{2}$. The signal to noise ratio (SNR) is defined as

$$
S N R=10 \log _{10} \frac{1}{\sigma_{w}^{2}}
$$

As performance measures, we use the averaged mean squared error (MSE) defined as

$$
\operatorname{MSE}\left(\hat{\theta}_{p}\right)=\frac{1}{M c} \sum_{i=1}^{M c}\left(\hat{\theta}_{p}^{(i)}-\theta_{p}\right)^{2}
$$

where $\hat{\theta}_{p}^{(i)}$ is the estimate of the $p$-th vibration signal parameter $\theta_{p}$ at the $i$-th run. The numerical values are obtained over $M c=100$ independent Monte Carlo trials where the SNR varies between -20 and $20 d B$ in all examples.

In the first example, we evaluate the performance of the proposed R-FQPT for the general QPS case. For that, we present a comparative study between the R-FQPT and the previously reviewed methods in section III. The simulated environment considers a single component and multiple component vibration signal with speed variation functions defined, in reduced frequency, as $f_{1}(t)=\left(0.02+\frac{0.00025}{2} t\right)$ and $f_{2}(t)=\left(0.045+\frac{0.00075}{2} t\right)$ and the complex amplitudes are $A_{1}=1 e^{j \pi / 10}$ and $A_{2}=1 e^{j \pi / 5}$. The signal is defined as

$$
x(t) \approx \sum_{p=1}^{P} A_{p} e^{j 2 \pi \int_{0}^{t} f_{p}(u) d u}
$$

with $P=1$ for single component and $P=2$ for multiple components case. The signal duration is about 256 samples.

The accuracy of the proposed R-FQPT algorithm is compared to the HAF method introduced by Barbarossa et al. in [18, 19], the iterative HAF method developed by Ikram et al. in [20], and the TFD method proposed by Barkat et al. in [16] as well as with the CRB given in section $\mathrm{V}$.

It can be seen in figure 1 that, the R-FQPT algorithm outperforms the HAF, the iterative HAF, and the TFD one for both single and multi-component signal cases, especially for low SNR. In the asymptotic region (higher SNR) the difference is very small but the proposed algorithm remains slightly better.

In the second example, we present a comparative study of the CRB expressions derived for general and order QPS cases. For that, we consider the signal models presented in 3 and 4 , where the parameters are $N=256, P=3$, $\rho=[1,1,1], \varphi=[\pi / 10, \pi / 5, \pi / 2.5], f=[0.1,0.2,0.3]$, $\gamma=[0.001,0.002,0.003]$ and $o=[1,2,3]$. From figure 2, it can be seen that there is almost no gain for the estimation of the amplitude parameters when considering the model in Eq. (4) instead of (3). However, for the estimation of the quadratic phase parameters, a gain of about $3 \mathrm{~dB}$ is observed when considering the additional information given by the O-QPS model. 

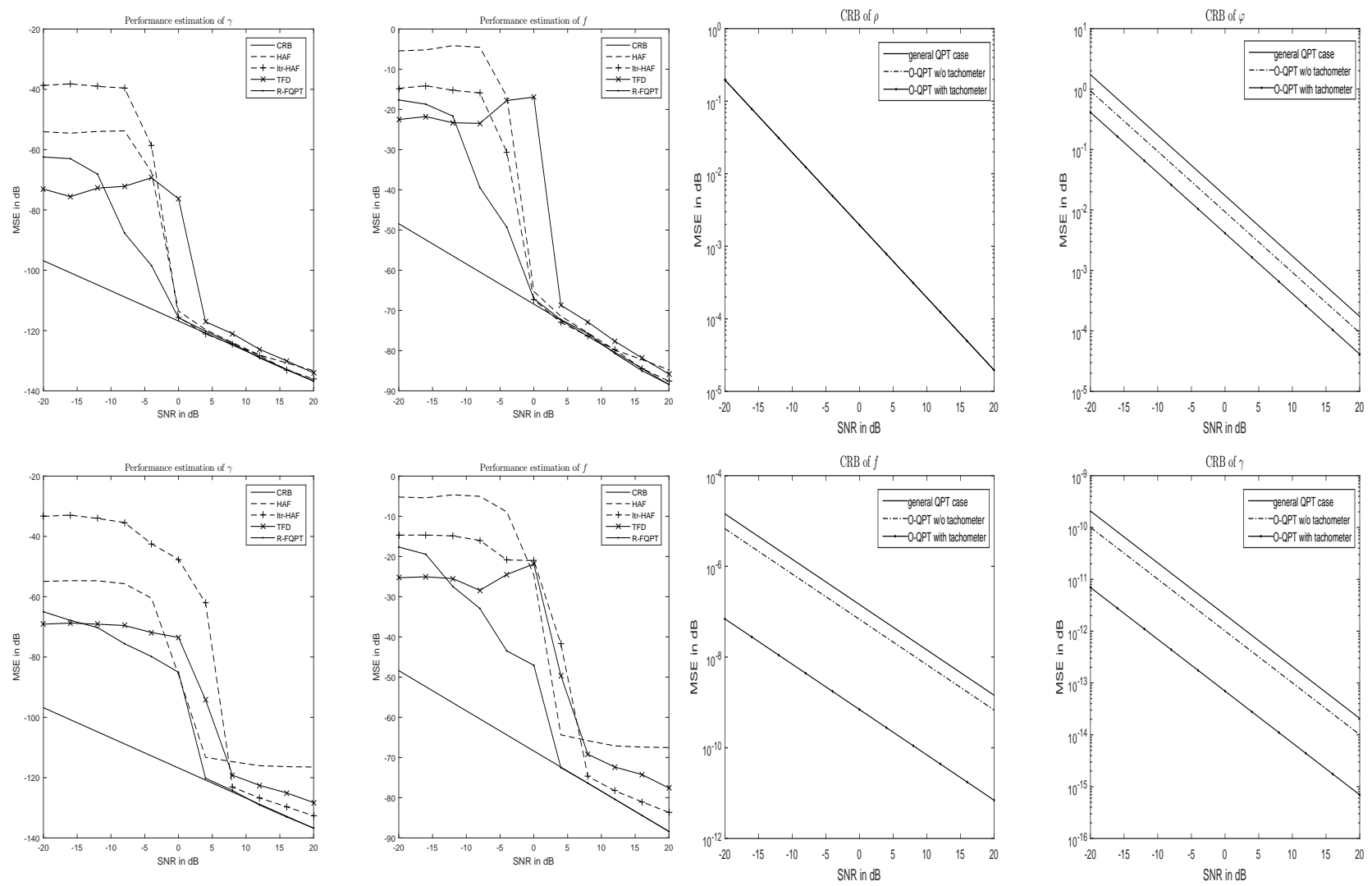

Fig. 1: MSE of $f$ and $\gamma$ versus SNR for a single component signal (top plots) and a two components signal (bottom plots).

Now, if a tachometer is used, the previous gain becomes much larger and exceeds $10 \mathrm{~dB}$ for the considered example. Consequently, when possible, the use of a tachometer for the vibration signal analysis is highly recommended.

In the third scenario, we evaluate the performance of the proposed Adapted-HAF method for the order QPS case with tachometer. The performance of the proposed method is compared to the corresponding derived CRBs in section V. For that, We consider the simulated multi-component signal of example 2. In figure 3, we compare the cumulated MSE of the 3 estimated orders with their corresponding CRB. As we can see, the MSE performance of the proposed adapted-HAF algorithm reaches the CRB for moderate SNR values (for $S N R>0 d B$ in the two components case and $S N R>5 d B$ for the three components case). Hence the Adapted-HAF presents a low cost alternative solution when the tachometer is available.

In the final experiment, we will apply the proposed methods to a time varying vibration of a Diesel engine. Results are compared to those of, Speed Transform (ST) proposed by Capdessus at al. in [7] and Velocity Synchronous-DFT developed by Borghesani et al. in [6].

R-FQPT is first compared to ST and VSDFT through a simulated scenario that considers a three component vibration signal with speed variation function defined, in reduced frequency, as $f_{r}(t)=(0.16+0.00003 t)$ given by tachometer. The signal is defined as

$$
x(t) \approx \sum_{p=1}^{3} A_{p} e^{j 2 \pi o_{p} \int_{0}^{t} f_{r}(u) d u} \approx \sum_{p=1}^{3} A_{p} e^{j 2 \pi o_{p}\left(0.16 t+\frac{0.00003}{2} t^{2}\right)}
$$

Fig. 2: CRB comparison

where $A_{1}=A_{2}=A_{3}=1, o_{1}=2, o_{2}=4$ and $o_{3}=6$. The signal comprises 512 samples.

It can be seen in figure 4 that the proposed approach allows estimating jointly the polynomial coefficient parameters of the rotation speed, that corresponds well to the considered harmonic's orders. Indeed, three corresponding peaks are located on a straight line whose direction vector corresponds to the reduced rotation frequency variations and the positions of the peaks allow estimating the corresponding order. R-FQPT thus allows estimating directly the parameters of the three chirp components whereas the ST and VSDFT give the order of the harmonic relatively to a known rotation speed.

\section{CONCLUSION}

The paper introduced a thorough analysis of the parameter estimation problem associated to variable speed vibration signal. The latter is shown to be a quadratic phase signal for which a plethora of estimation algorithms exist in the literature.

After a brief survey of the major class of methods, we proposed two new solutions, namely the R-FQPT for the general QPS case and the Adapted HAF for the O-QPS.

Simulation experiments have been conducted to illustrate the behavior and advantages of the proposed solutions and compare their performance to those of the major algorithms from the literature. We have also derived the CRB expressions for the different quadratic phase models and exploited them to investigate the potential gain due to the side information related to the Order QPS model and to the tachometer use, respectively.

As a perspective, we believe it would be highly beneficial to 

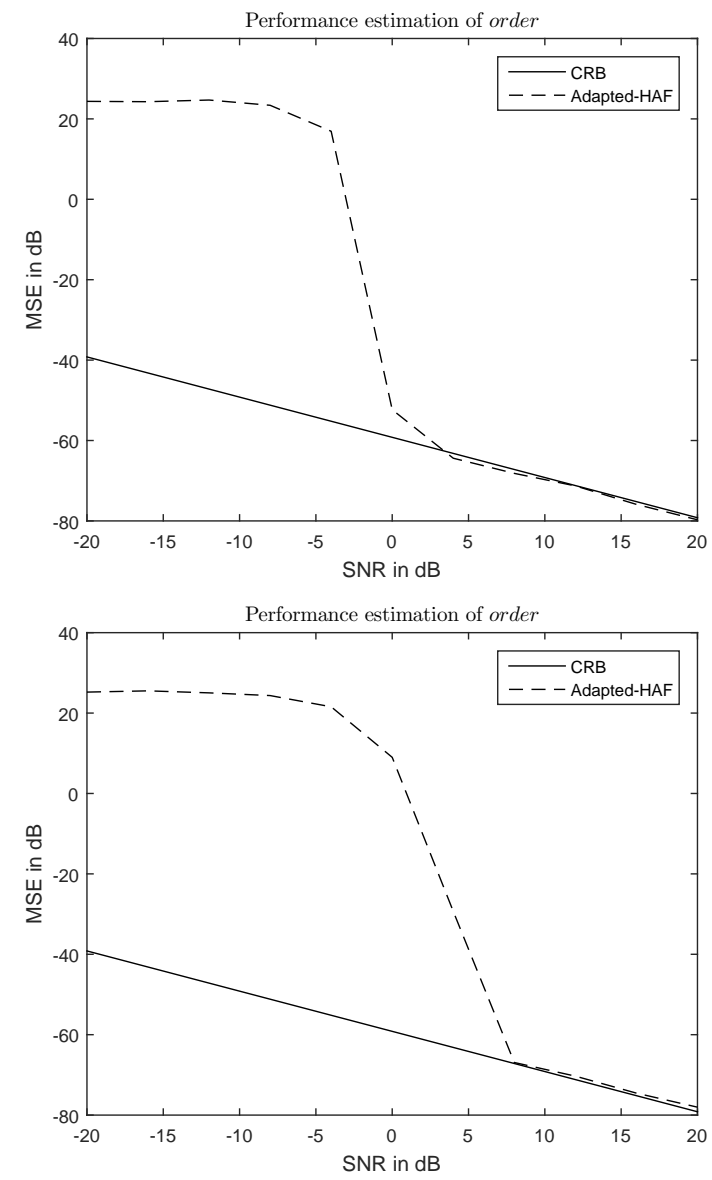

Fig. 3: MSE of the estimated order versus SNR for a two components signal (top plots) and a three components signal (bottom plots)

consider in future works an extension of our paper dedicated to an enlarged comparative study of all methods (QPT, HAF, ST, TVDFT, VSDFT) that can be used for the considered vibration signal analysis. In addition, an enriched validation and testing of these methods, based on real data measurements, would be of high importance for their practical use.

\section{REFERENCES}

[1] N. O. Myklestad, Fundamentals Of Vibration Analysis, Paperback edition, 2018.

[2] C. Scheffer, Practical Machinery Vibration Analysis and Predictive Maintenance, Newnes Publication, 2004.

[3] J. R. Blough, D. L. Brown, H. Vold, The time variant discrete Fourier transform as a tracking order method, Proceedings of Society of Automotive Engineers Noise and Vibration Conference, SAE Paper Number 972006, 3 (54) (1997) 823-837. https://doi.org/10.4271/972006

[4] J. R. Blough, Development and analysis of time variant discrete Fourier transform order tracking, Mechanical Systems and Signal Processing, 6 (17) (2003) 185-1199. https://doi.org/10.1006/mssp.2002.1500

[5] P. Borghesani, P. Pennacchi, R. B. Randall, R. Ricci, Order tracking for discrete-random separation in variable speed conditions, Mechanical Systems and Signal Processing, (30) (2012) 1-22. https://doi.org/10.1016/j.ymssp.2012.01.015

[6] P. Borghesani, P. Pennacchi, S. Chatterton, R. Ricci, The velocity synchronous discrete Fourier transform for order tracking in the field of rotating machinery, Mechanical Systems
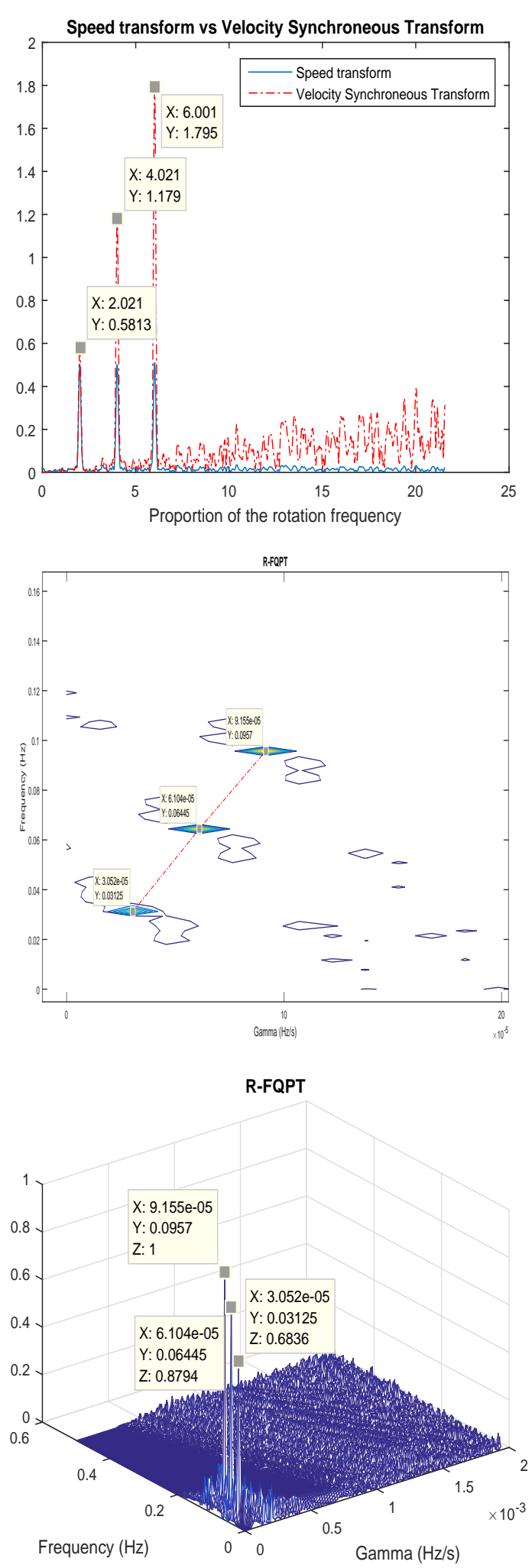

Fig. 4: ST, VSDFT and R-FQPT of a simulated vibration signal (function of reduced frequency) 
and Signal Processing, 44 (2014) 118-133. https://doi.org/ 10.1016/j.ymssp.2013.03.026

[7] C. Capdessus, E. Sekko, J. Antoni, Speed transform, a new time-varying frequency analysis technique, Advances in Condition Monitoring of Machinery in Non-Stationary Operations, Lecture Notes in Mechanical Engineering, (2014) 23-35. https://doi.org/10.1007/978-3-642-39348-8_2

[8] J. Roussel, M. Haritopoulos, E. Sekko, C. Capdessus, J. Antoni, Application of speed transform to the diagnosis of a roller bearing in variable speed, in Proc. of Surveillance 7, Chartres, France, Oct. 2013 (October 2013).

[9] J. R. Blough, A survey of DSP methods for rotating machinery analysis, what is needed, what is available, Journal of Sound and Vibration, (262) (2003) 707-720. http://doi.org/10.1016/S0022460X(03)00118-4

[10] P. Borghesani, R. Ricci, S. Chatterton, P. Pennacchi, A new procedure for using envelope analysis for rolling element bearing diagnostics in variable operating conditions, Mechanical Systems and Signal Processing, (38) (2013) 23-35. https://doi.org/10.1016/j.ymssp.2012.09.014

[11] P. O'Shea, M. Farquharson, G. Ledwich, Estimation of timevarying mains frequencies, in Proc. of the Australasian Universities Power engineering Conference (2003).

[12] V. Katkovnik, Nonparametric estimation of instantaneous frequency, IEEE Transactions on Information Theory, 43 (1) (1998) 183-189. https://doi.org/10.1109/18.567676

[13] J. Angeby, Estimating signal parameters using the nonlinear instantaneous least squares approach, IEEE Transactions on Signal Processing, 48 (10) (2000) 2721-2732. https://doi.org/10.1109/78.869022

[14] A. Abbadi, C. Capdessus, K. Abed-Meraim, E. Sekko, Vibration signal parameters estimation in variable speed using quadratic phase transform, in Proc. of Surveillance 8, Roanne, France, Oct. 2015.

[15] A. Abbadi, K. Abed-Meraim, C. Capdessus, A. Belouchrani, F. Soltani, Localisation en champ proche par formation de voies: Algorithme rapide et analyse de résolution, in Proc. GRETSI 2015, Lyon, France, Sep. 2015 (Sep. 2015).

[16] B. Barkat, K. Abed-Meraim, Algorithms for blind components separation and extraction from the time-frequency distribution of their mixture, EURASIP Journal of Applied Signal Processing (JASP), (13) (2004) 2025-2033. https://doi.org/10.1155/S1110865704404193

[17] B. Barkat, K. Abed-Meraim, A blind components separation procedure for FM signal analysis, in Proc. of ICASSP, USA, (2) (2002) 1425-1428. https://doi.org/10.1109/ICASSP.2002.5745820

[18] S. Barbarossa, A. Scaglione, G. Giannakis, Product high-order ambiguity function for multicomponent polynomial-phase signal modelling, IEEE Transactions on Signal Processing, 46 (3) (1998) 691-708. https://doi.org/10.1109/78.661336

[19] S. Barbarossa, V. Petrone, Analysis of polynomial-phase signals by the integrated generalized ambiguity function, IEEE Transactions on Signal Processing, 45 (2) (1997) 316-327. https://doi.org/10.1109/78.554297

[20] M. Z. Ikram, K. Abed-Meraim, Y. Hua, Iterative parameter estimation of multiple chirp signals, Electronics Letters, 33 (8) (1997) 657-659. https://doi.org/10.1049/el:19970466

[21] M. Z. Ikram, K. Abed-Meraim, Y. Hua, Estimating the parameters of chirp signals: An iterative approach, IEEE Transaction on Signal Processing, 46 (12) (1998) 3436-3441. https://doi.org/10.1109/78.735320
[22] M. Z. Ikram, K. Abed-Meraim, Y. Hua, Fast quadratic phase transform for estimating the parameters of multicomponent chirp signals, Digital Signal Processing, 7 (2) (1997) 127-135. https://doi.org/10.1006/dspr.1997.0286

[23] Y. Ju, G. Bi, Generalized fast algorithms for the polynomial timefrequency transform, IEEE Transaction on Signal Processing, 55 (2007) 4907-4915. https://doi.org/10.1109/TSP.2007.896102

[24] M. Z. Khan, M. Suleman, M. Ashraf, A. Q. Khan, Some practical aspects of balancing an ultra-centrifuge rotor, Journal of Nuclear Science and Technology, 24 (11) (1987) 951-959. https://doi.org/10.1080/18811248.1987.9733526

[25] L. F. Villa, A. Reñones, J. R. Perán, L. J. de Miguel, Statistical fault diagnosis based on vibration analysis for gear test-bench under non-stationary conditions of speed and load, Journal of Mechanical Systems and Signal Processing, 29 (May 2012) 436446. https://doi.org/10.1016/j.ymssp.2011.12.013

[26] J. Drouet, Diagnostic qualité de boîtes de vitesse automatiques à partir de mesures acoustiques et vibratoires, VIbrations, SHocks and NOise (VISHNO), Le Mans, France, (2016) 2441-2446.

[27] M. Desbazeille, Diagnostic de groupes électrogènes diesel par analyse de la vitesse de rotation du vilebrequin, Thèse de doctorat de l'Université Jean Monnet, (2011, (HAL Id: tel-00563111 https://tel.archives-ouvertes.fr/tel-00563111)).

[28] A. C. Sall, Comportement dynamique d'un redresseur de turbomachine aéronautique: Effets du désaccordage, Thèse soutenue à l'Ecole Centrale de Lyon le 8 Décembre, (2011).

[29] E. Swanson, C. D. Powell, S. Weissman, A practical review of rotating machinery critical speeds and modes, Journal of Sound and Vibration, 39 (5) (2005) 10-17.

[30] S. Peleg, B. Porat, B. Friedlander, The discrete polynomial transform (DPT), its properties and applications, IEEE in Proc. TwentyFifth Asilomar Conf Signals, Systems and Computers, 1 (1991) 116-120. https://doi.org/10.1109/ACSSC.1991.186425

[31] S. Peleg, B. Porat, Estimation and classification of signals with polynomial phase, IEEE Trans. Inform. Theory, 37 (1991) 422-430. https://doi.org/10.1109/18.75269

[32] B. Porat, Digital Processing of Random Signals, Theory and Methods, Vol. sec. 12, NJ: Prentice-Hall, Englewood Cliffs, 1994.

[33] A. Pinkus, Weierstrass and approximation theory, Journal of Approx. Theory, 107 (1) (2000) 1-66. https://doi.org/10.1006/jath.2000.3508

[34] K. Abed-Meraim, L. Nguyen, V. Sucic, F. Tupin, B. Boashash, An image processing approach for underdetermined blind separation of nonstationary sources, in Proc. of Int. Symp. on Sig. and Image Proc. and Analysis, Rome, (Sep. 2003) 185-1199. https://doi.org/10.1109/ISPA.2003.1296856

[35] Z. Feng, M. Liang, F. Chu, Recent advances in time-frequency analysis methods for machinery fault diagnosis: A review with application examples, Journal of Mech. Syst. Signal Process. 38 (1) (2013) 165-205. https://doi.org/10.1016/j.ymssp.2013.01.017

[36] O. Cardona-Morales, L. Avendaño, G. Castellanos-Domínguez, Nonlinear model for condition monitoring of non-stationary vibration signals in ship driveline application, Journal of Mechanical Systems and Signal Processing, 44 (1-2) (2014) 134-148. https://doi.org/10.1016/j.ymssp.2013.08.029

[37] P. O'Shea, On refining polynomial phase signal parameter estimates, IEEE Transactions On Aerospace and Electronic Systems, 46 (3) (2010) 978-987. https://doi.org/10.1109/TAES.2010.5545168 
Ali Abbadi was born in 1979 in Rouiba, Algeria. He received the State Engineering Degree in Electrical Engineering in 2003 and the Magistère degree in 2008 in signal processing, both from Ecole Militaire Polytechnique, Algeria. He completed his $\mathrm{PhD}$ degree in 2018 with a specialization in radar detection and array signal processing which is his current field of research.

Cécile Capdessus was born in France in 1965. She received an engineering degree and a $\mathrm{Ph}$. $\mathrm{D}$. in signal processing from the Institut National Polytechnique de Grenoble (INPG), in Grenoble, France, in 1989 and 1992 respectively. After a one year post-doctoral position at the Universitat Politècnica de Catalunya (UTC), in Barcelona, Spain, she spent two years as an assistant lecturer first at the University of Orléans and then at the INPG. Since 1995 she has worked as a lecturer at the University of Orléans, and is currently a member of PRISME Laboratory. Her main research field is the application of signal processing techniques to vibration analysis.

Karim Abed-Meraim was born in 1967, Algiers, Algeria. He received the State Engineering Degree from Ecole Polytechnique, Palaiseau, France, in 1990, the State Engineering Degree from Ecole Nationale Supérieure des Télécommunications (ENST), Paris, France, in 1992, the M.Sc. degree from Paris XI University, Orsay, France, in 1992 and the Ph.D. degree from the ENST in 1995 (in the field of Signal Processing and communications). From 1995 to 1998, he took a position as a research staff at the Electrical Engineering Department of the University of Melbourne where he worked on several research project related to "Blind System Identification for Wireless Communications", "Blind Source Separation", and "Array Processing for Communications", respectively. From 1998 to 2012 he has been Assistant then Associate Professor at the Signal and Image Processing Department of Telecom-ParisTech. His research interests are in signal processing for communications, adaptive filtering and tracking, array processing and statistical performance analysis. In september 2012 he joined the University of Orléans (PRISME Lab.) as a full Professor. He is the author of over 480 scientific publications including book chapters, patents, and international journal and conference papers. Dr. Abed-Meraim is an IEEE Fellow, an IEEE SAM-TC member, a member of the TAC on Signal Processing for Multisensor Systems (EURASIP), and a senior area editor for the IEEE Transactions on Signal Processing.

Edgard Sekko was born in Cotonou, Benin, in 1971. He received the M.Sc. and the doctoral degree in electrical engineering from Claude Bernard University, Lyon, France, in 1993 and 1998, respectively. Since 1999, he has been with the Technology Institute of Engineering Science at the University of Orleans, France, as an Assistant Professor. His main research activities interest vibration analysis applied to both the diagnosis of gearbox and rolling bearing in variable speed by using $H \alpha$ estimation theory. 